\title{
Porosity Calculation of Tight Sand Gas Reservoirs with GA-CM Hybrid Optimization Log Interpretation Method
}

\author{
Ya-Nan Duan1, Bao-Zhi Pan1, Xue Han', Hai-Tao Zhang3, Xiao-Ming Yang3 \\ ${ }^{1}$ College of Earth Exploration Science and Technology, Jilin University, Changchun, China \\ ${ }^{2}$ Shengli Petroleum Engineering Co. Ltd., the First Branch of the Logging Company, Dongying, China \\ ${ }^{3}$ Exploration and Development Institute of Changqing Oilfield, PetroChina, Xi'an, China \\ Email: 116025147@qq.com
}

Received March 2014

\section{Abstract}

Tight sand gas reservoirs are our country's fairly rich unconventional natural gas resources, and their exploration and development is of prime importance. Sulige Gas Field which located in the northern Ordos Basin is tight sand gas reservoirs. It is typically featured by low porosity and low permeability, and the error of porosity calculation by traditional methods is larger. Multicomponent explanation model is built by analyzing the thin slice data, and the objective function is got according to the concept of optimization log interpretation method. This paper puts the Genetic Algorithm and the Complex Algorithm together to form the GA-CM Hybrid Algorithm for searching the optimal solution of the objective function, getting the porosity of tight sandstone gas reservoirs. The deviation got by this method is lesser compared with the core porosity, with a high reliability.

\section{Keywords}

Porosity, Tight Sand Gas Reservoirs, Low Porosity and Low Permeability, GA-CM, Optimization, Multicomponent Explanation Model

\section{Introduction}

The porosity data is not only important for calculating oil saturation of reservoirs, for the estimation of oil, gas and other resources, but also important for the country's future plan of development. In recent years, the tight sandstone gas reservoirs have become a major force of natural gas supply in our country (Kang \& Luo, 2007). By the end of 2011, the natural gas reserve that China has explored is $3.38 \times 10^{12} \mathrm{~m}^{3}$, accounting for $49.5 \%$ of the total explored reserves of large gas fields throughout the country, the annual production of $222.5 \times 108 \mathrm{~m}^{3}$, accounting for $24.6 \%$ in 2011 China’s total natural gas (Cao, Zhao, Liu, Hu, Fan, \& Wang, 2013). The low porosity and strong heterogeneity of the tight sandstone reservoirs, make the calculated porosity deviations with conventional methods larger, comparing with the core porosity. Therefore, considering a variety of factors, rea- 
sonable mathematical methods need to be used in order to obtain a more accurate porosity of tight sandstone reservoirs prediction models.

The optimization log interpretation method is a method processing the synthesis with a variety of well logging information, and is an effective way to evaluate complex oil and gas reservoirs. Since the 1980s, optimization log interpretation method technology has developed and improved. In the process of algorithm research, a large number of domestic and foreign scholars make a lot of efforts in the mathematical method selection criterion, the improvements of classical methods, the developments of new algorithms (Yong, Sun, \& Bi, 1988; Xiao, 1989; Zou, Wei, Chai, Han, \& Zhang, 1999). Among them, the Genetic Algorithm and its improved algorithms for their unique advantages are widely used. In addition to its own algorithm improvements, Genetic Algorithm can also be combined with other algorithms to constitute a new algorithm. Han Xue et al. (2012) combine Genetic Algorithm with complex algorithm constituting GA-CM hybrid algorithm to process glutenite reservoirs, both accurately and efficiently searching the optimal solution, having achieved good results in sandstone, pebbly sandstone and glutenite intervals. This paper applied GA-CM hybrid optimization log interpretation method to the SuliIge tight sandstone reservoirs, and reconstructed the multicomponent model in accordance with the characteristics of tight sandstone, getting the porosity and every mineral content volume of tight sandstone reservoirs, having achieved good effects. This method can be extended to the tight sandstone gas reservoirs.

This paper regarded tight sandstone gas reservoirs of $\mathrm{P}_{2} \mathrm{~h}$ and $\mathrm{P}_{1} \mathrm{~s}$ interval of Permian in eastern Sulige Area as the research objectives. The lithological composition of $\mathrm{P}_{2} \mathrm{~h}$ and $\mathrm{P}_{1} \mathrm{~s}$ interval in this area is complex. It is mainly composed of lithic quartz sandstone, lithic sandstone and a small amount of quartz sandstone (Cheng, Song, Jing, He, Zhang, \& Duan, 2013).

\section{The Realization of GA-CM Hybrid Optimization Log Interpretation Method}

\subsection{The Establishment of the Multicomponent Model}

To create the optimization log interpretation mathematical model, the reservoir multi-component model needs to be determined. 4831 thin section analysis data of the objective area was collated, and the pie chart of rock composition and clastic composition of $\mathrm{P}_{2} \mathrm{~h}$ and $\mathrm{P}_{1} \mathrm{~s}$ interval in eastern Sulige gas field (Figure 1, Figure 2) were drawn. It can be found from Figure 1, Figure 2 that the reservoirs consist of clastics and interstitial material, and clastic constituent is mainly quartz, followed by detritus, very little feldspar. Interstitial material is mainly composed of gyulekhite, kiesel and kaolinite. To simplify the model, this paper assumes that the pore fluid is only water. A multi-component model is established in Figure $\mathbf{3}$ for the studied area, considering that the reservoirs consist of four parts: water, shale, quartz and detritus.

\subsection{The Eestablishment of the Mathematical Model}

The building of the optimization log interpretation mathematical model is the basis of the optimization log interpretation, including the establishment of the objective function and the constraints. According to the principle

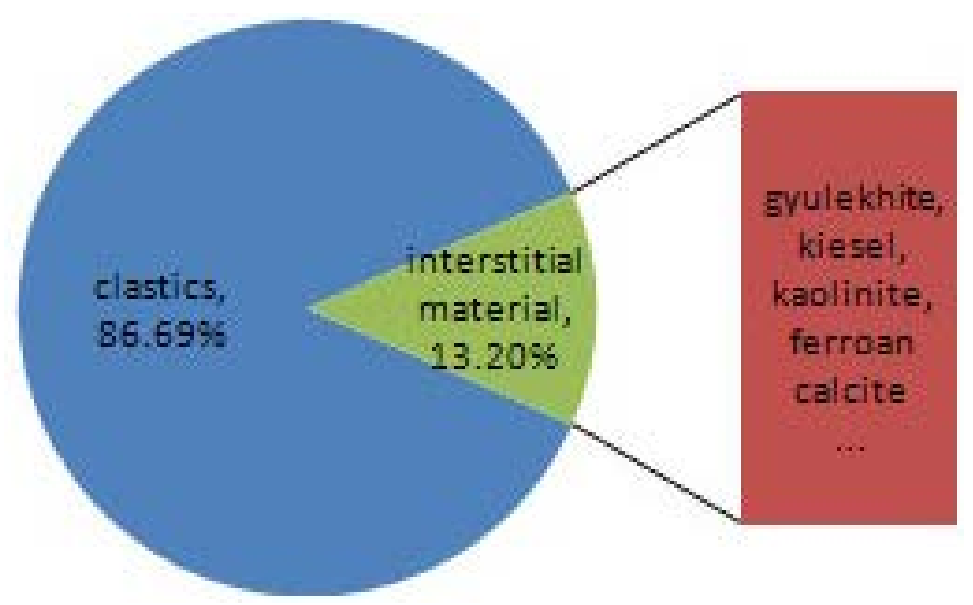

Figure 1. The pie chart of rock composition. 


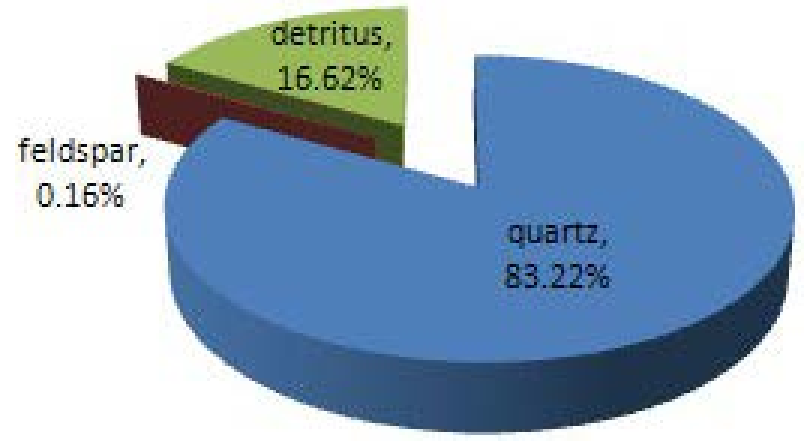

Figure 2. The pie chart of clastic composition.

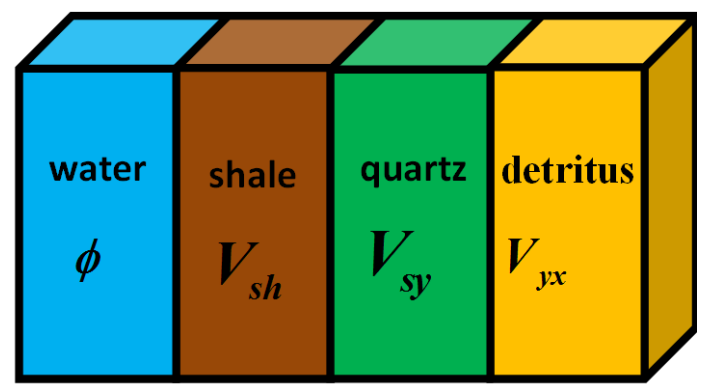

Figure 3. The multi-component model schematic diagram.

of non-linear weighted least squares method, the objective function of the optimization interpretation is established as Equation (1).

$$
\min F(x, a)=\min \sum_{i=1}^{m} \frac{\left[a_{i}-f_{i}(x, z)\right]^{2}}{\sigma_{i}^{2}+\tau_{i}^{2}}
$$

where, $x$ is the unknown parameter of the reservoirs; $a_{i}$ is the actual log response value; $\sigma_{i}$ is the measurement error of the log methods during the measurement process; $\tau_{i}$ is the response equation error which are induced by the model. $f_{i}(x, z)$ is the log response equation. This paper selected three logging curves including CNL, DEN, AC to calculate, and their log response equations are shown by the following matrix, as Equation (2).

$$
\left[\begin{array}{c}
C N L \\
D E N \\
A C
\end{array}\right]=\left[\begin{array}{cccc}
C N L_{f} & C N L_{\text {shale }} & C N L_{\text {quartz }} & C N L_{\text {detritus }} \\
D E N_{f} & D E N_{\text {shale }} & D E N_{\text {quartz }} & D E N_{\text {derritus }} \\
A C_{f} & A C_{\text {shale }} & A C_{\text {quartz }} & A C_{\text {derritus }}
\end{array}\right] \cdot\left[\begin{array}{c}
\phi \\
V_{\text {shale }} \\
V_{\text {quartz }} \\
V_{\text {detritus }}
\end{array}\right]
$$

After establishing the objective function, the constraints also need to be defined to ensure the reservoir parameters obtained have practical significances. Its constraints are as Equation (3).

$$
\left\{\begin{array}{l}
0 \leq \phi, V_{\text {shale }}, V_{\text {quartz }} 、 V_{\text {detritus }} \leq 1 \\
\phi+V_{\text {shale }}+V_{\text {quartz }}+V_{\text {detritus }}=1
\end{array}\right.
$$

\subsection{The Selection of the Geologic Parameters in Tight Sandstone}

The log response value of shale component can be got by drawing the neutron-density crossplot and the acoustic wave-density crossplot. The detritus is mainly composed of metamorphic rock, igneous rock and a little sedimentary rock. The main components of the metamorphic detritus are phyllite, metamorphic sandstone, schist, slate and so on; the igneous detritus are mainly composed of the effusive and cryptocrystalline rock. The parameters of each component are determined by seeking the main mineral logging characteristics sheet (Yong, 1996) and the main igneous rock log response characteristics (Oberto, 1984). The logging parameters for each 
component in the studied area are shown in Table 1. These explanation parameters are taken in Equation (1) to obtain the minimum of the objective function, so the unknown reservoir parameters can be got.

\subsection{The Basic Principle of GA-CM Hybrid Algorithm}

The optimization log interpretation is based on the generalized geophysical inversion theory, applying optimization mathematical algorithm, statistical probability and error theory as well as quality control techniques to process a synthesis with a various of log information, getting the reservoir parameters which reflect the underground complex lithology formations original appearance for the reservoir evaluation, oil and gas analysis (Yong, 1996). In this paper, GA-CM Hybrid Algorithm is adopted for solving the objective function optimal solution. The following are the basic principles of Genetic Algorithm and Complex Algorithm.

The Genetic Algorithm is based on Darwin's natural selection theory and Mendel's Segregation Law to iterate to find the optimal solution. As is shown in Figure 4, in the Genetic Algorithm, first a set of initial population is randomly generated and the fitness of each individual is calculated, and then the first generation is randomly generated and the optimization criterion of the first generation is verified. If the optimization criterion is not met, then by selection, crossover and mutation of three genetic operators, the new individuals can be generated and the fitness of the new individual can be calculated. The off spring who replace their parents, are inserted into the population to constitute a new generation. Iterate as mentioned above until meeting up optimization criterion (Cui, 2010).

Figure 5 is the schematic diagram of Complex Method. In the Complex Algorithm, a complex shape with $\mathrm{k}$ vertices in an n-dimensional space firstly needs to be created, and the objective function value of each vertex of the Complex shape would be calculated. The bad point with the maximum value of the objective function should be removed, and the mapping point of the removed dead pixel with the center of the rest points as the mapping center is search for. The previous bad point is replaced with the mapping point to constitute a new complex shape. By iterating constantly in this way, the complex shaped shrinks constantly. When the centroid of each

Table 1. The parameter datasheet of skeleton mineral components.

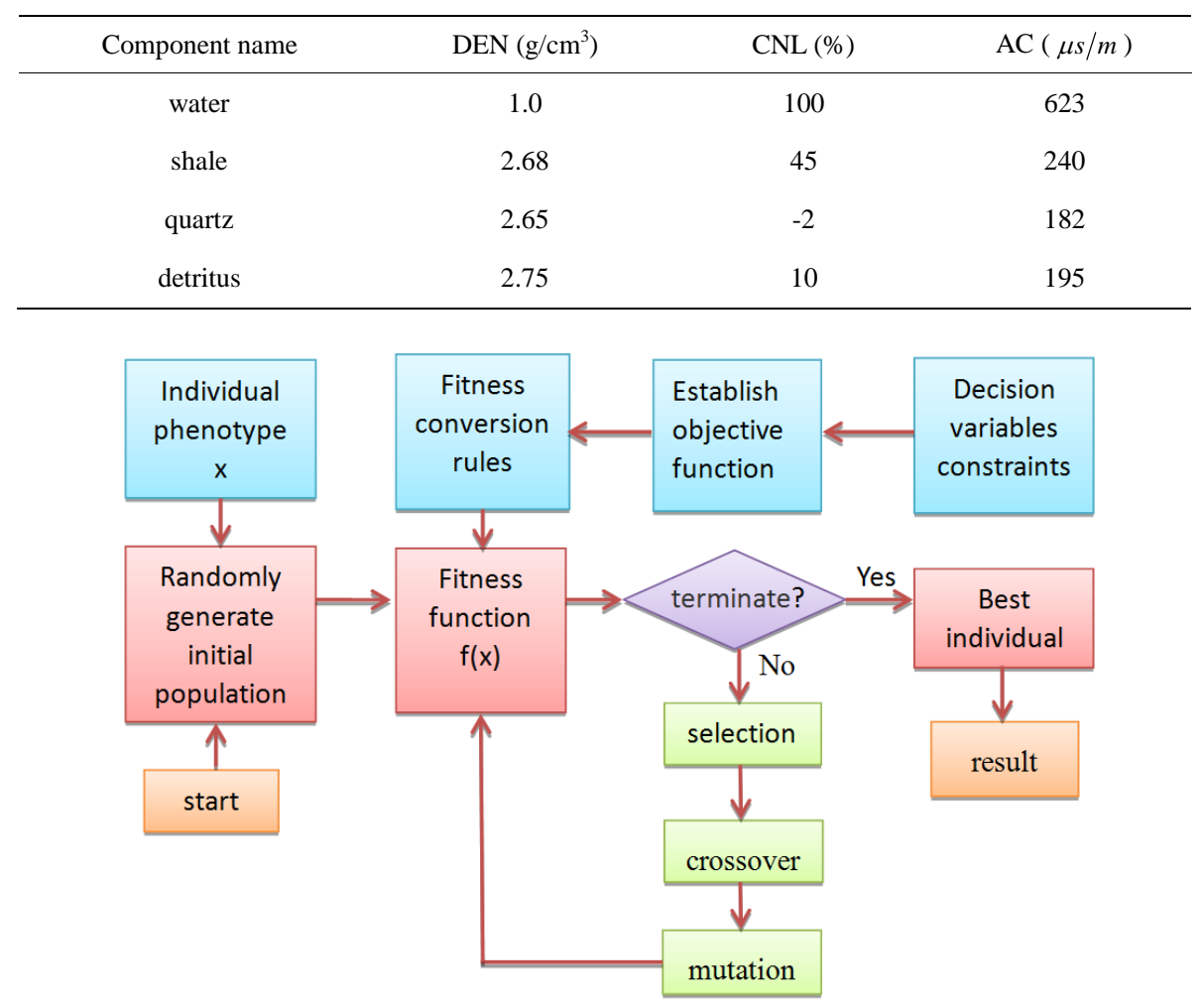

Figure 4. The flowchart of Genetic Algorithm to solve function optimization problems. 
complex shape vertex is sufficiently close to each vertex, the iteration stops and then the point with the minimum objective function value of the complex shape vertices is output as the optimal solution.

Although the Genetic Algorithm has strong global search capability, it easily converges. So this paper proposed a method of combining the Complex Method which has a strong local search ability with Genetic Algorithm to form a Hybrid Genetic Algorithm. The results calculated by the Genetic Algorithm are used as the initial vertices of the Complex Algorithm. Both of the two algorithms showed their strengths respectively, thus improved the efficiency and ensured the accuracy of the optimal solution.

\section{GA-CM Method for Solving Reservoir Parameters}

Zhao70 well $2653 \mathrm{~m}$ - $2673 \mathrm{~m}$ and zhao52 well $2725 \mathrm{~m}$ - $2750 \mathrm{~m}$ were respectively processed by the program of GA-CM hybrid optimization log interpretation method. The results of two well sections are shown respectively in Figure 6 and Figure 7. Compared with the core porosity, the calculated porosity average absolute errors are $0.61 \%$, and $1.37 \%$. The average relative errors are $6.57 \%$ and $15.9 \%$. The data showed a high accuracy. There-

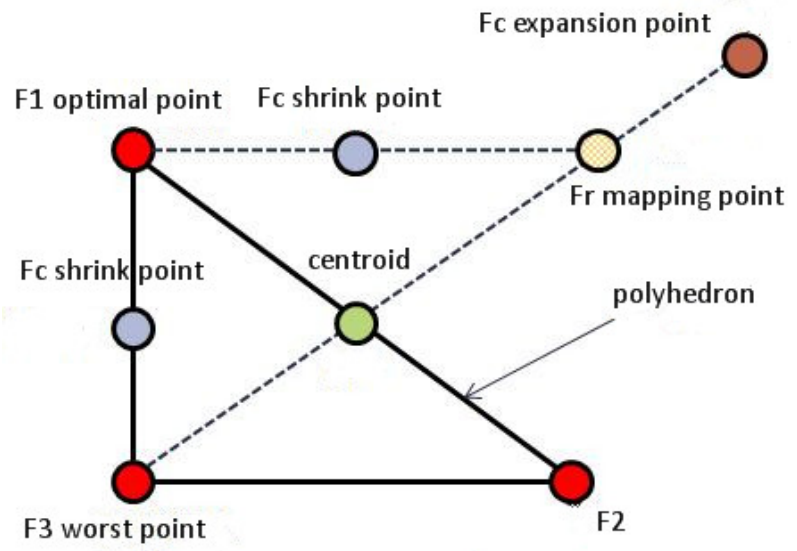

Figure 5. The complex algorithm principle sketch map.

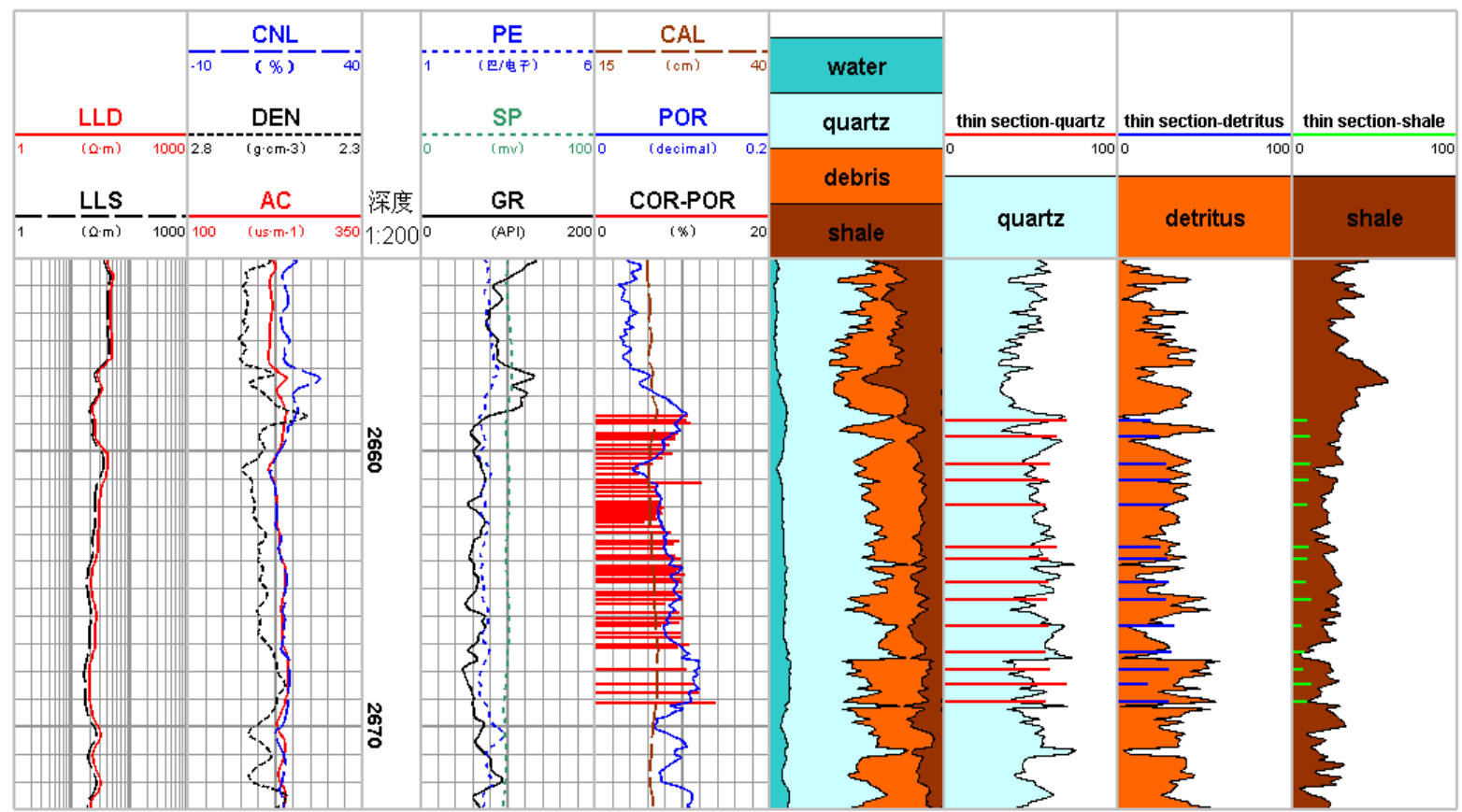

Figure 6. Zhao70 optimization log interpretation method result figure. 


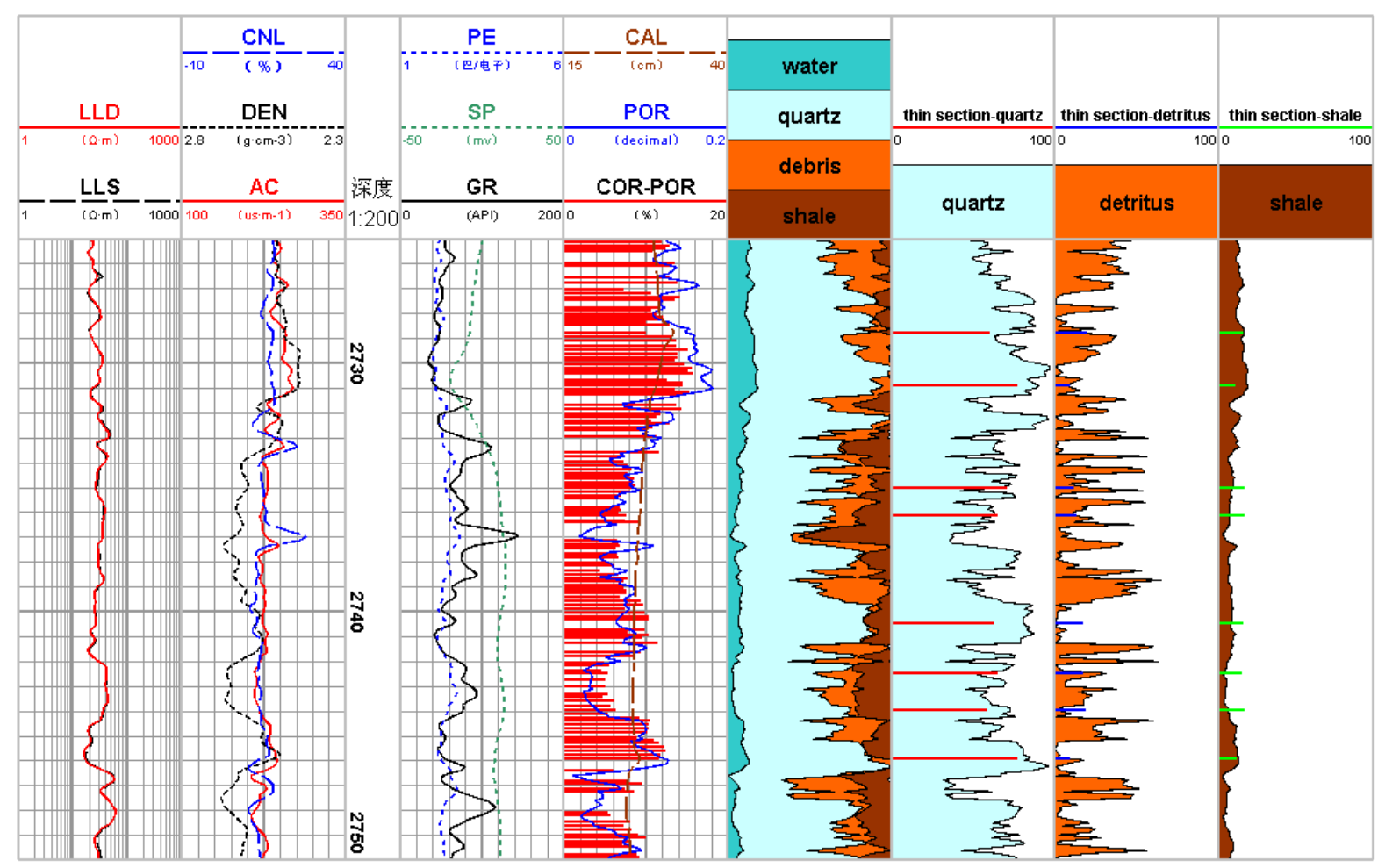

Figure 7. Zhao52 optimization log interpretation method result figure.

fore, the accuracy of the porosity of tight sandstone with GA-CM hybrid optimization log interpretation method is higher and it can reach the standard of practical application.

\section{Conclusion}

1) The lithology of Sulige tight sandstone reservoirs is complex, including quartz, detritus. The detritus components are diverse and the content of each component changes in a wide range. In particular, detritus is also radioactive, having a great influence on the calculation of the shale content. Various factors should be considered in the establishment of the reservoir model, so multi-component model is adopted, in order to obtain more accurate reservoir parameters.

2) The Genetic Algorithm is a kind of heuristic method and is applied extensively these years. However, the Genetic Algorithm easily converges. In order to overcome this shortcoming, this paper proposed a method of combining the Complex Method with GA and formed a Hybrid Genetic Algorithm. It has been proved that GA-CM hybrid optimization algorithm could find the optimal solution with high efficiency.

3) The porosity, shale content and other parameters of the tight sandstone reservoir were calculated by the GA-CM hybrid optimization log interpretation method. Compared with the core data, the error is small and with a higher reliability. Therefore, this method is suitable for obtaining the porosity tight of the sandstone reservoir.

\section{References}

Cao, Q., Zhao, J., Liu, X., Hu, A., Fan, L., \& Wang, H. (2013). Determination of Physical Property Limits for the Gas Accumulation in Tight Sandstone Reservoirs in the Eastern Ordos Basin. Acta Petrolei Sinica, 34, 1040-1048.

Cheng, Z., Song, Z., Jing, C., He, Y., Zhang, L., \& Duan, Q. (2013). Tight Reservoir Diagenetic Reservoir Facies Classification and Log Responses in East Sulige Area. Well Logging Technology, 37, 154-161.

Cui, S. (2010). Some Improvements of the Genetic Algorithm and Their Applications. Master's Degree Thesis, Hefei: University of Science and Technology of China.

Han, X. (2012). Research on Glutenite Reservoirs with GA-CM Hybrid Optimization Log Interpretation Method in Lishu Fault Depression. Master’s Degree Thesis, Changchun: Jilin University. 
Kang, Y., \& Luo, P. (2007). Current Status and Prospect of Key Techniques for Exploration and Production of Tight sand Stone Gas Reservoirs in China. Petroleum Exploration and Development, 34, 239-245.

Oberto, S. (1984). Fundamentals of Well-log Interpretation: The Acquisition of Logging Data. Amsterdam: Elsevier Publishers.

Xiao, L. (1989). Evaluation for the Result Obtained from Optimal Well Logging Interpretation. Geophysical Prospecting for Petroleum, 28, 64-71.

Yong, S., Sun, J., \& Bi, Z. (1988). Evaluation of Complex Reservoirs Using Optimization Methods. Well Logging Technology, 12, 18-28.

Yong, S. (1996). Optimization Log Interpretation. Beijiing: Petroleum University Press.

Zou, C., Wei, Z., Chai, X., Han, C., \& Zhang, H. (1999). Optimization of Log Interpretation Using Genetic Algorithm. Well Logging Technology, 23, 361-365. 